\title{
Biochemical and Cellular Mechanism of Protein Kinase CK2 Inhibition by Deceptive
}

\section{Curcumin}

Giorgio Cozza $^{1 *}$, Francesca Zonta ${ }^{2 *}$, Andrea Dalle Vedove ${ }^{3}$, Andrea Venerando ${ }^{4}$, Roberto Battistutta $^{5}$, Maria Ruzzene ${ }^{2}$, Graziano Lolli $^{3}$

Author Affiliations:

${ }^{1}$ Department of Molecular Medicine, University of Padua, Via U. Bassi 58/B, 35131 Padua, Italy

${ }^{2}$ Department of Biomedical Sciences and CNR Institute of Neuroscience, University of Padua, Via U. Bassi 58/B, 35131 Padua, Italy

${ }^{3}$ Centre for Integrative Biology, University of Trento, via Sommarive 9, 38123 Povo - Trento, Italy

${ }^{4}$ Department of Comparative Biomedicine and Food Science, University of Padua, Viale dell'Università 16, 35020 Legnaro (PD), Italy

${ }^{5}$ Department of Chemical Sciences, University of Padua and Institute of Biomolecular Chemistry, National Research Council (CNR), Via Marzolo 1, 35131 Padua, Italy

* The authors contributed equally to the work

Correspondence to Roberto Battistutta: roberto.battistutta@unipd.it, Maria Ruzzene: maria.ruzzene@unipd.it and Graziano Lolli: graziano.lolli@unitn.it.

\section{ABSTRACT}

Protein kinase CK2 is an anti-apoptotic cancer-sustaining protein. Curcumin, reported previously as a CK2 inhibitor, is too bulky to be accommodated in the CK2 active site and rapidly degrades in solution generating various ATP-mimetic inhibitors; with a detailed comparative analysis, by means of both protein crystallography and enzymatic inhibition, ferulic acid was identified as the principal curcumin-degradation product responsible for CK2 inhibition. The other curcumin derivatives vanillin, feruloylmethane and coniferyl aldehyde are weaker CK2 inhibitors. The high instability of curcumin in standard buffered solutions flags this compound, which is included in many commercial libraries, as a possible source of misleading interpretations, as was the case for CK2. Ferulic acid does not show any cytotoxicity and any inhibition of cellular CK2, due to its poor cellular permeability. However, curcumin acts as a prodrug in the cellular context, by conveying its degradation products inside the treated cells, thus rescuing CK2 inhibition and consequently inducing cell death. Through the intracellular release of its degradation products, curcumin is expected to affect various target families; here we identify the first bromodomain of BRD4 as a new target for those compounds. 


\section{Introduction}

CK2 is a Ser/Thr protein kinase able to phosphorylate hundreds of substrates and involved in diverse cellular processes from apoptosis to differentiation [1]. It has been proposed that it is kept inactive by an unusual self-inhibitory assembly into high order oligomeric states and its activity restored upon necessity [2-4]. Although not being an oncogene, its activity has been found elevated in various cancers, especially haematological malignancies [5-7]. Its anti-apoptotic role has been associated with its involvement in sustaining cancer growth and in protecting cancerous cells. A number of small molecules interfering with CK2 activity have been developed over the last years culminating in the CK2 inhibitor CX-4945 (silmitasertib), currently in phase II clinical trials for the treatment of various tumours, and granted the Orphan Drug status for cholangiocarcinoma in the USA [8-10].

Curcumin (Fig. 1a) is a diarylheptanoid used as food additive for flavouring and colouring, in dietary supplements and in cosmetics; clinical trials were conducted or are ongoing for a wide variety of pathological conditions [11]. Curcumin at $10 \mu \mathrm{M}$ concentration was reported to reduce CK2 activity by about $75 \%$ [12]. However, it has also been shown that curcumin undergoes very rapid degradation in aqueous solution and the reliability of the numerous claims about the diverse biological activities of curcumin has been recently object of much debate [13-16]. Here we demonstrate that curcumin is not a CK2 inhibitor by itself and that the observed inhibition is exerted by the curcumin degradation products vanillin, feruloylmethane, ferulic aldehyde and, most predominantly, ferulic acid (Fig. 1a). Similarities between the biological activities reported for these compounds and some of those attributed to curcumin can be identified. Ferulic acid has been proposed as a potential treatment for Alzheimer's disease, cancer and cardiovascular diseases [17]. Among other activities, vanillin was found to induce apoptosis in cancer cells by inhibiting NF- $\kappa \mathrm{B}$ phosphorylation and activation [18]. Feruloylmethane (dehydrozingerone, vanillylidene acetone) has a broad range of reported biological activities among which antioxidant, anticancer and antiinflammatory activities [19]. Ferulic aldehyde (coniferyl aldehyde) has been reported to have 
cytostatic or cytotoxic activity in various cancer cells and to overcome the resistance of U373 glioblastoma cells to pro-apoptotic stimuli [20].

Bromodomains are acetylated-lysines (Kac) recognising modules, generally associated with additional domains involved in chromatin remodelling and transcriptional regulation [21]; bromodomains have crucial epigenetic implications in cancer, inflammation and neurological disorders, with a number of inhibitors in clinical trials [22]. Various compounds, which share a similar chemical organization with curcumin degradation products, were recently reported as micromolar inhibitors of BET (Bromodomain and Extra-Terminal motif proteins: BRD2, BRD3, BRD4 and BRDT) bromodomains [23-24]. Ferulic acid is shown here to bind in the Kac pocket of the first BRD4 bromodomain. Notably, BRD4 activity is regulated by CK2 [25] and the combination of CX-4945 with the BET inhibitor JQ1 (also currently in clinical trials) revealed superior to single agent treatments in T-cell acute lymphoblastic leukemia and fragile $\mathrm{X}$ syndrome [26-27].

\section{Results}

Crystallographic studies of curcumin and its degradation products in complex with CK2

Curcumin has been reported as a CK2 inhibitor [12]. However, using our docking protocol specifically validated on CK2, we only obtained unconvincing poses for curcumin ((1E,6E)-1,7bis(4-hydroxy-3-methoxyphenyl)hepta-1,6-diene-3,5-dione, Fig. 1a) bound to CK2 (data not shown). X-ray crystallographic studies were performed to inquire into this discrepancy. Curcumin was soaked for 24 hours in apo CK2 $2 \alpha$ crystals. Diffraction data analysis resulted in a positive electron density in the kinase ATP-binding pocket corresponding to roughly half of the curcumin molecule. Curcumin has been reported to be unstable in aqueous solution, with about $90 \%$ becoming degraded in 30 minutes in various products, identified as trans-6-(4'-hydroxy-3'methoxyphenyl)-2,4-dioxo-5-hexenal, ferulic aldehyde, ferulic acid, feruloylmethane, and vanillin [13]. The observed electron density was compatible with the last four compounds; therefore, each 
of them was tested separately in soaking experiments and their structures in complex with CK2 determined by X-ray crystallography (Fig. 1a).

Feruloylmethane (4-(4-hydroxy-3-methoxyphenyl)-3-buten-2-one) is oriented with the guaiacol group in the deepest part of the cavity (Fig. 1b). The hydroxyl group forms hydrogen bonds with side-chain nitrogen of Lys68, main-chain nitrogen of Asp175 and the conserved water W1, with the methoxy oxygen also in H-bond contact with Lys68 side-chain nitrogen. While feruloylmethane is in hydrophobic and van der Waals contact with residues Leu45, Val53, Val66, Ile95, Phe113, Val116, Met163 and Ile174, no additional polar interactions are formed with the hinge region. Buried surface area is $335 \mathrm{~A}^{2}$, corresponding to $87.6 \%$ of feruloylmethane surface area, as calculated by PISA [28].

Ferulic aldehyde ((E)-3-(4-hydroxy-3-methoxyphenyl)prop-2-enal) (Fig. 1c) and vanillin (4Hydroxy-3-methoxybenzaldehyde) (Fig. 1d) bind to CK2 $\alpha$ with an orientation superimposable to that of feruloylmethane. As a consequence, polar interactions with the deepest part of the cavity are replicated, while overall interface areas are reduced due to the smaller size of the two aldehydes with respect to the ketone. This reduction is limited in the case of ferulic aldehyde, missing a single methyl group as compared to feruloylmethane (interface area $312 \mathrm{~A}^{2}, 86.9 \%$ of its surface). The smallest molecule instead, vanillin (MW $152.15 \mathrm{~g} / \mathrm{mol}$ ), has an interface area of $278 \mathrm{~A}^{2}, 90.8 \%$ of its solvent accessible area; it is the only one that does not contact residues on the opposite side of the cavity, i.e. Leu45 and Val116. However, vanillin is the most acidic molecule with the lowest $\mathrm{pKa}$ for the guaiacol hydroxyl (7.8, as compared with 9.5 for both feruloylmethane and ferulic aldehyde). 


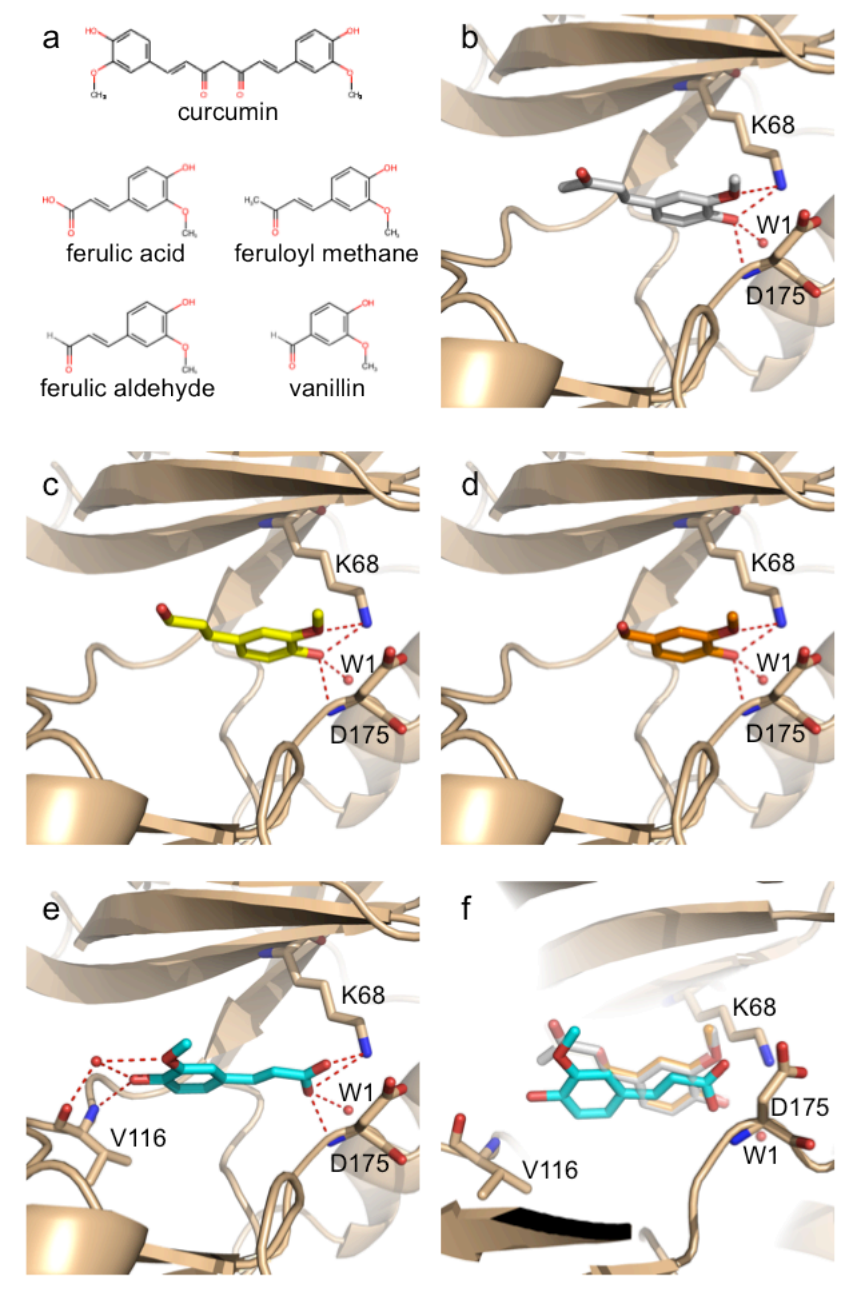

Figure 1. CK2 alpha in complex with curcumin degradation products. a) Structural formulae of compounds soaked in CK2 alpha crystals. b) Feruloylmethane (white) bound to CK2 alpha ATP-pocket (gold); hydrogen bonds are shown as red dashed lines. c) Ferulic aldehyde (yellow) and d) vanillin (orange) adopt essentially the same pose observed with feruloylmethane. e) Ferulic acid (cyan) binds with opposite orientation in respect to the other curcumin degradation products. f) Electron density in curcumin-soaked crystals is interpreted as generated by different amounts of each of the four degradation products. Colour code is same as above and opacity for the compounds has been assigned according to their relative occupancy.

Ferulic acid ((E)-3-(4-hydroxy-3-methoxy-phenyl)prop-2-enoic acid) binds to CK2 $\alpha$ with opposite orientation with respect to the three molecules described above, that is with the guaiacol group pointing toward the hinge region and the carboxylic function located in the positively charged area (Fig. 1e). This last interacts with side-chain nitrogen of Lys68, main-chain nitrogen of Asp175 and the conserved water W1. On the other side, the guaiacol hydroxyl is in hydrogen bond contact with 
Val116 main-chain nitrogen. Moreover, both guaiacol oxygens form a water-mediated hydrogen bond with Val116 main chain carbonyl. Buried surface area is $338 \mathrm{~A}^{2}$, similar to feruloylmethane, corresponding to $91.2 \%$ of its surface. However, among curcumin derivatives, ferulic acid is the only compound in polar contact with both the hinge region and the Lys68 positively charged area. We translated binding poses of all four compounds in the crystal structure of CK2 $\alpha$ soaked with curcumin (see methods). The observed electron density is adequately fitted with a mixture of $46 \%$ ferulic acid, 30\% feruloylmethane, 22\% vanillin and 2\% ferulic aldehyde (Fig. 1f). Despite the low occupancy for the ferulic aldehyde, that makes questionable its presence in the curcumin-soaked crystal, the structure of this compound bound to CK2 shown above indicates its ability to effectively bind to CK2.

\section{Biochemical characterization of curcumin and derivatives}

Curcumin and its derivatives were assayed as CK2 inhibitors (Fig. 2 and Table 1), keeping the wellknown CK2 inhibitor quinalizarin as internal control [29-30]. After $30 \mathrm{~min}$ of incubation at $37^{\circ} \mathrm{C}$, ferulic acid resulted to be the most potent and ATP-competitive inhibitor of this series with an $\mathrm{IC}_{50}$ value of $0.84 \mu \mathrm{M}(\mathrm{Ki}=0.41 \mu \mathrm{M}$, Fig. $2 \mathrm{~d})$; the aldehyde (ferulic aldehyde) and ketone (feruloylmethane) derivatives of ferulic acid resulted to have $\mathrm{IC}_{50}$ values almost two orders of magnitude higher (40.1 $\mu \mathrm{M}$ and $65.5 \mu \mathrm{M}$, respectively). Similarly, to ferulic aldehyde and feruloylmethane, vanillin shows an $\mathrm{IC}_{50}$ value in the high micromolar range $(53.4 \mu \mathrm{M})$. In these experimental conditions, curcumin inhibitory activity $\left(\mathrm{IC}_{50}=2.38 \mu \mathrm{M}\right)$ is intermediate between that of ferulic acid and the ones of its derivatives, and compatible with its CK2 inhibitory effect reported in literature and measured in a similar experimental setting [12]. Quinalizarin inhibitory activity $\left(\mathrm{IC}_{50}=0.11 \mu \mathrm{M}\right)$ is consistent with values available from literature [29-30].

Compounds were also tested at the shorter incubation times of 15 and 5 minutes (Fig. $2 \mathrm{~b}$ and 2a). Curcumin inhibitory activity decreases by shortening the incubation time with $\mathrm{IC}_{50}$ values of 10.8 $\mu \mathrm{M}$ at 15 minutes and $85.1 \mu \mathrm{M}$ at 5 minutes (Table 1); all other compounds present similar activity 
profiles independently from the incubation time. These results clearly suggest that the CK2 inhibitory activity of curcumin strictly depends in vitro on its degradation to ferulic acid.

Table 1. $\mathrm{IC}_{50}$ values of curcumin and derivatives for $\mathrm{CK} 2 \alpha_{2} \beta_{2}$ at different incubation times (min).

\begin{tabular}{lccc}
\hline Compound & $\mathbf{I C}_{\mathbf{5 0}}(\boldsymbol{\mu M}), \mathbf{t}=\mathbf{3 0} \mathbf{~ m i n}$ & $\mathbf{I C}_{\mathbf{5 0}}(\boldsymbol{\mu M}), \mathbf{t}=\mathbf{1 5} \mathbf{~ m i n}$ & $\mathbf{I C}_{\mathbf{5 0}}(\boldsymbol{\mu M}), \mathbf{t}=\mathbf{5} \mathbf{~ m i n}$ \\
\hline Ferulic Acid & $0.84 \pm 0.10(\mathrm{Ki}=0.41)$ & $0.81 \pm 0.08$ & $0.87 \pm 0.05$ \\
Ferulic aldehyde & $40.1 \pm 1.81$ & $40.9 \pm 1.94$ & $40.6 \pm 2.21$ \\
Feruloylmethane & $65.5 \pm 3.26$ & $68.6 \pm 4.15$ & $61.8 \pm 3.22$ \\
Vanillin & $53.4 \pm 2.91$ & $52.2 \pm 1.87$ & $57.6 \pm 2.32$ \\
Curcumin & $2.38 \pm 0.15$ & $10.8 \pm 0.64$ & $85.1 \pm 3.11$ \\
Quinalizarin & $0.11 \pm 0.03$ & $0.15 \pm 0.04$ & $0.13 \pm 0.02$ \\
\hline
\end{tabular}

a
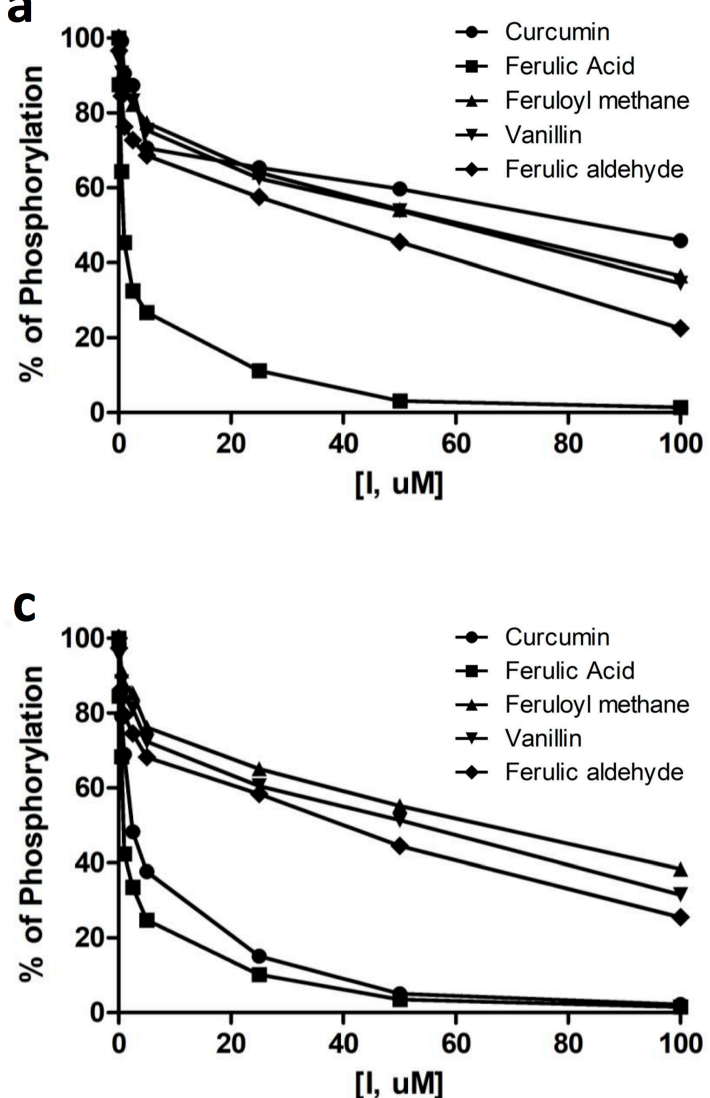

b
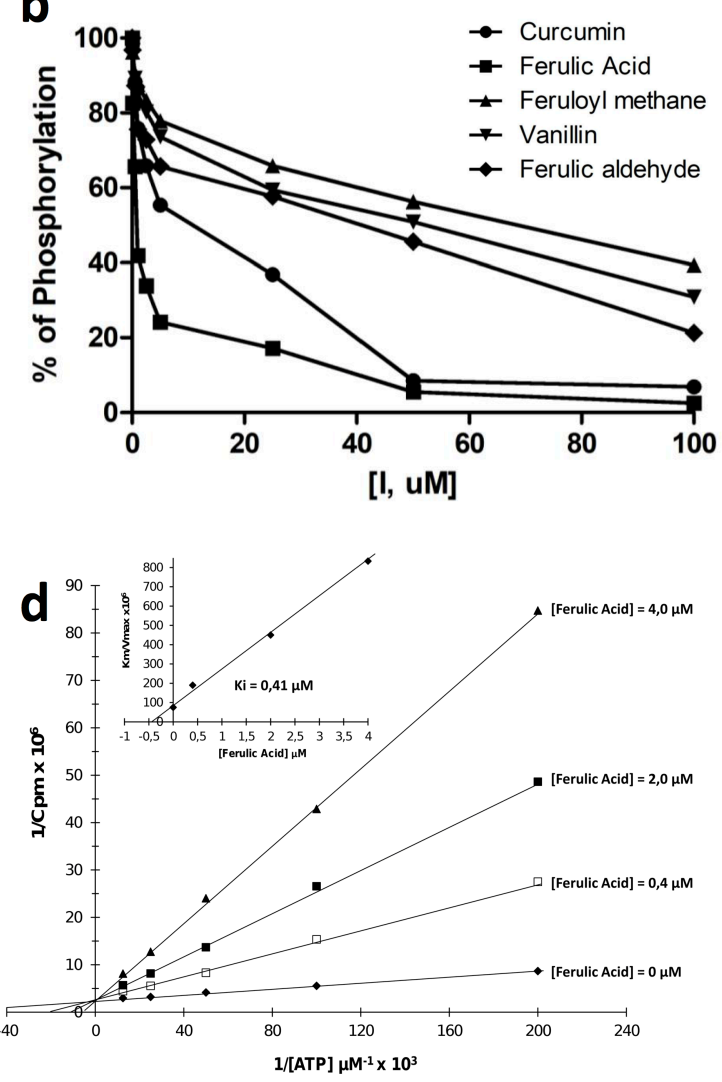

Figure 2. CK2 inhibition by curcumin and its degradation products. IC50 curves at incubation times of 5 (a), 15 (b) and 30 (c) minutes. ATP-competitive CK2 inhibition by ferulic acid is consistent with its binding in the kinase ATP-pocket observed in the crystallographic structure (d). 


\section{In cell activity of curcumin and ferulic acid}

Cell treatment with curcumin has been previously reported to affect CK2 activity [31-32]; however, our results suggested that the effective inhibitor of CK2 upon cell treatment with curcumin could be its derivative ferulic acid, as already observed with the purified protein. To check this hypothesis, Jurkat cells were incubated with increasing concentrations of ferulic acid, and analysed for major effects on cell viability (Fig. 3), as expected when CK2 is inhibited in cancer cells [33]. Cells were totally insensitive to ferulic acid up to $100 \mu \mathrm{M}$ (Fig 3a); consistently, when we measured CK2 activity in treated cells, we did not observe any inhibition (Fig 3c and 3e), while the well-known CK2 inhibitor CX-4945 [34], used as control at $5 \mu \mathrm{M}$ concentration, induced cell death and a drastic reduction of CK2 activity. Similar results were obtained by treatment of other cancer cell lines, namely SK-N-BE and SH-SY5Y (data not shown). We interpreted the inefficacy of the negatively charged ferulic acid as due to its poor cell permeability.
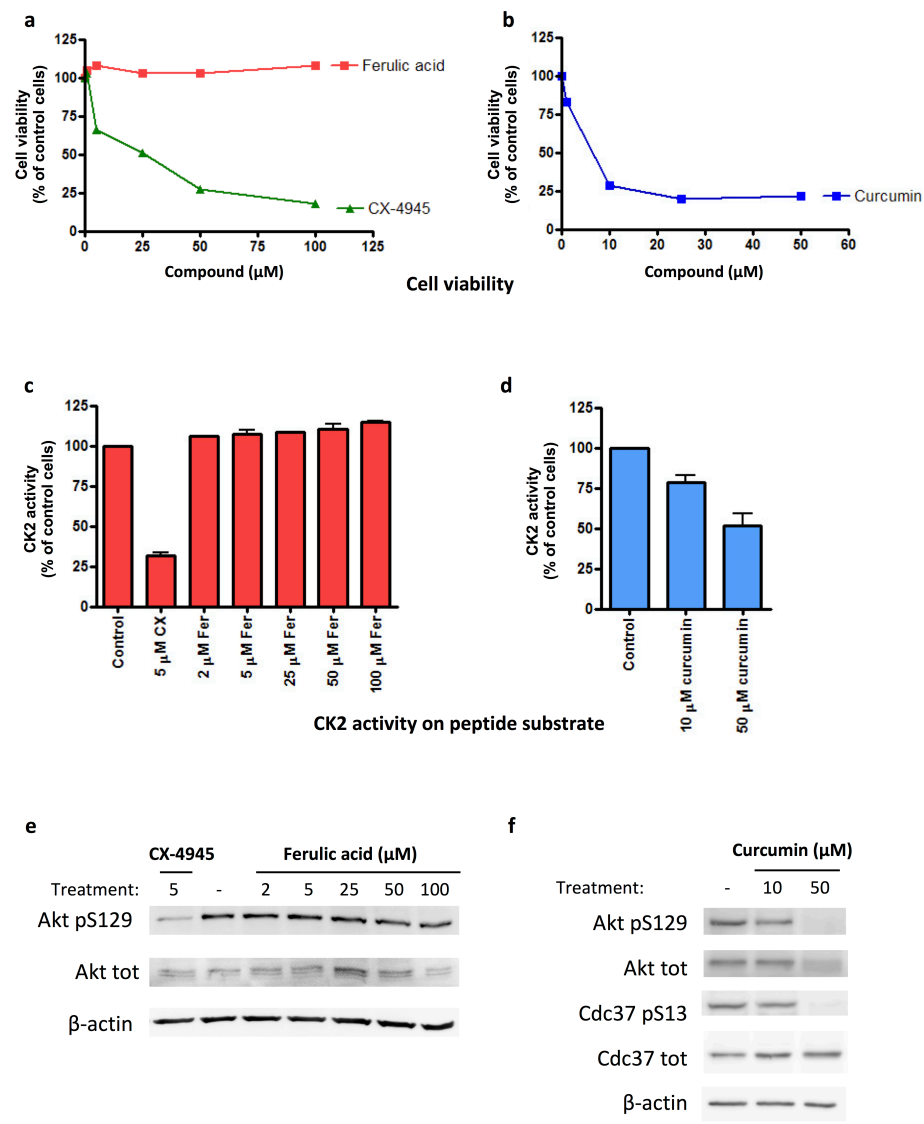

CK2 activity on endogenous substrates 
Figure 3. Effects of ferulic acid and curcumin in cells. Top panel: Jurkat cells were treated for $24 \mathrm{~h}$ with increasing concentrations of ferulic acid or CX-4945 (a), or of curcumin (b). Cell viability was assessed by the MTT method and is shown as $\%$ of vehicle-treated (Control) cells (mean values \pm SE of three experiments). Middle panel: CK2 activity on CK2-tide peptide substrate was measured using 1-2 $\mu \mathrm{g}$ proteins from total lysate of Jurkat cells treated for $5 \mathrm{~h}$ with ferulic acid or CX-4945 (c), or with curcumin (d); CX: CX-4945; Fer: ferulic acid. Activity is reported as \% of control cells (mean values \pm SE of three experiments). Lower panel: Jurkat cells were treated with ferulic acid or CX-4945 (e), or with curcumin (f) for $5 \mathrm{~h} .20 \mu \mathrm{g}$ of proteins from total lysate were analysed by WB with the indicated antibody. $\beta$ actin WB (5 $\mu \mathrm{g}$ proteins) was used as loading control. Representative WB of at least two independent experiments are shown.

In contrast, curcumin induced major cell death in the same experimental settings at a concentration as low as $10 \mu \mathrm{M}$ (Fig 3b), as similarly reported by others [35]. Concomitantly, we observed significant CK2 inhibition either by assessing CK2 activity in lysates from treated cells (Fig. 3d), or by evaluating the phosphorylation state of the CK2 endogenous targets Akt Ser129 [36] and Cdc37 Ser13 [37] (Fig. 3f). The effects of curcumin on cell viability and CK2 activity were comparable to those obtained with the CK2 inhibitor CX-4945 (Fig. 3). Our results in cells, in combination with those obtained in vitro with recombinant CK2, suggest that the action of curcumin on endocellular CK2 is exerted by conveying its degradation products inside the cells, thus allowing the effective CK2 inhibitor ferulic acid to reach its intracellular target.

\section{Ferulic acid binds in the Kac pocket of the BRD4 first bromodomain}

Owing to the similarity of ferulic acid with previously reported BET bromodomains inhibitors [2324], the compound was soaked into apo crystals of the BRD4 first bromodomain. The guaiacol group binds in the deepest part of the pocket with the hydroxyl group recapitulating the interactions of the acetyl group of Kac with the protein matrix; two hydrogen bonds are formed between the guaiacol hydroxyl and the evolutionary conserved Asn140 and Tyr97 (water-bridged for this last, Fig. 4a). The phenyl ring is sandwiched between side chains of the gatekeeper residue Ile146 and 
Val87. The acrylic acid moiety runs in between the ${ }^{81} \mathrm{WPF}^{83}$ shelf and the tip of the ZA loop (Leu92), establishing a number of hydrophobic and van der Waals contacts with this apolar regions; the carboxylate group is located at the entrance of the cavity in a complex water network connecting it to the protein matrix (Gln85 main and side chains and main chains of Pro82 and Asp88, Fig. 4b).

Comparison of the structures of the BRD4 first bromodomain in complex with ferulic acid and acetylated peptides shows that the guaiacol methoxy points in the direction of the aliphatic part of the Kac residue, which then suggests the growing direction for improving potency of ferulic acid as a BET inhibitor (Fig. 4b).
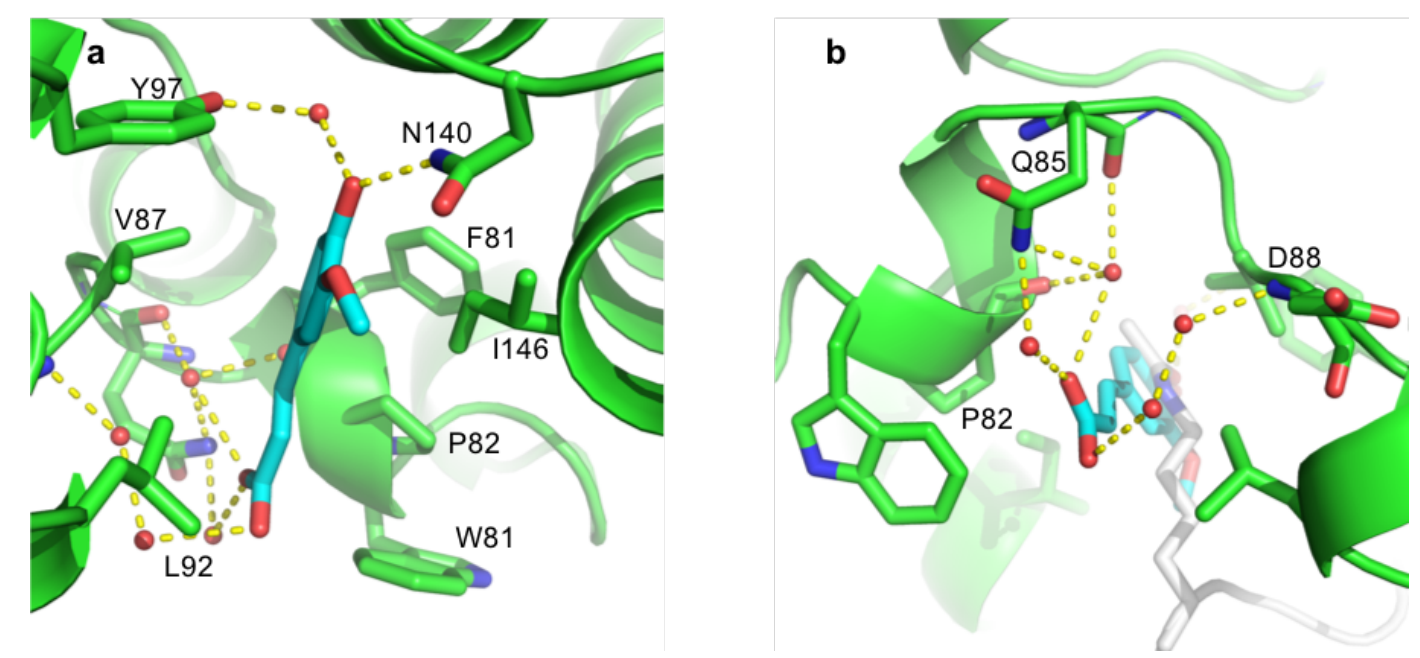

Figure 4. Binding mode of ferulic acid to the Kac pocket of the BRD4 first bromodomain. Two different views are shown, one highlighting interactions for the guaiacol group (a), the other centred on the acrylic acid moiety (b). BRD4 is shown in green, ferulic acid in cyan and the Kac peptide (from PDB 3UVW) in white.

\section{Discussion}

The CK2 inhibitory profiles of curcumin (time-dependent) and of its four derivatives (timeindependent, Fig. 2 and Table 1), supported by the respective crystal structures in complex with the kinase (Fig. 1), show that the in vitro CK2 inhibitory effect of curcumin is due to its degradation products, mainly to ferulic acid. 
The binding modes of the four curcumin degradation products share a common feature: the most acidic atom is placed in the CK2 positively charged region, as also observed with other inhibitors [38]. This atom is the guaiacol hydroxyl oxygen for vanillin, feruloylmethane and ferulic aldehyde but the carboxyl oxygen in ferulic acid. As such, ferulic acid is rotated of about $180^{\circ}$ with respect to the other molecules; this noticeable difference is even more striking considering the single nonhydrogen substitution between ferulic acid and feruloylmethane.

Ferulic acid has a very good ligand efficiency (LE, defined as the binding energy per non-hydrogen atom (HA) of a ligand [39-40]) of $0.61 \mathrm{kcal} \mathrm{mol}^{-1} \mathrm{HA}^{-1}$; by comparison, the CK2 inhibitor CX4945, currently in clinical trials, with an IC50 $=1 \mathrm{nM}$ has $\mathrm{LE}=0.50 \mathrm{kcal} \mathrm{mol}^{-1} \mathrm{HA}^{-1}$. These data highlight the relevance of ferulic acid as a CK2 inhibitor and as an excellent scaffold for further modifications. This compound has been proposed as a potential therapeutic agent for different pathologies [41]. However, in our hands it appears to be very poorly effective when directly administrated to cancer cells. A possible explanation for this discrepancy is that observations of cellular effects reported by others as directly induced by ferulic acid are due to actions played at the membrane level, on receptors or other molecules not requiring cellular penetration, as in the case of the inhibition of melanoma cell proliferation and the blockage of the PI3K-Akt pathway [42]. This prompts further investigation to assess the potential of ferulic acid as direct inhibitor of ecto-CK2, a fraction of CK2 localized at the external side of plasma membrane, which has been shown not to affect cellular proliferation, but to promote migration of cancerous cells then enhancing their invasive and metastatic potential [43]. In this context, additional interest is provided by diseasesustaining roles recently proposed for ecto-CK2 also in Alzheimer and parasitic disease [44-45].

Instead, when cell treatment is performed with curcumin, cellular viability and CK2 activity are heavily affected (Fig. 3), suggesting its ability to permeate membranes and generate ferulic acid inside the cells, thus acting as a prodrug in the cellular context.

The combined inhibition of CK2 and BRD4 with the respective inhibitors CX-4945 and JQ1 was shown to be more effective than the single agent treatments [26-27]. Ferulic acid is an inhibitor of 
both proteins and candidates as a valuable scaffold for the development of a BET-CK2 dual inhibitor. In this respect, the guaiacol methoxy could be further derivatized to complement the CK2 hinge $/ \alpha \mathrm{D}$ region and the $\mathrm{BRD} 4 \mathrm{ZA}$ and $\mathrm{BC}$ loops, as well as to increase lipophilicity and membrane permeability.

Finally, vanillin configures as a very interesting fragment for growing permeable inhibitors exploiting the interaction of the guaiacol moiety with the positively charged region of the CK2 ATP-binding pocket. Vanillin has approximately the same inhibitory potency observed for the larger compounds in the same orientation (ferulic aldehyde and feruloylmethane) and, as a consequence, a significantly better $\mathrm{LE}=0.54 \mathrm{kcal} \mathrm{mol}^{-1} \mathrm{HA}^{-1}$. This is due to the lower $\mathrm{pKa}$ of vanillin, a feature that would have to be maintained or even improved when designing new CK2 inhibitors based on this scaffold.

In conclusion, our results support the view of Shen and Ji [13] and warn against the risk of cursory attributing inhibitory activity to curcumin against various protein targets. This issue could have been seriously underestimated and has been recently object of debate [14-16]. Biological effects of curcumin should be evaluated with care, especially considering its inclusion in various commercial screening libraries; as in the case of $\mathrm{CK} 2$, the observed activity could be spuriously attributed to curcumin. The vast number of in vitro biological activities reported for curcumin is most probably the sum of those exerted by a mixture of molecules, some of them configuring as fragments with promiscuous binding capability.

\section{Materials and Methods}

Protein production, crystallization and structure solution

CK2 alpha was produced as previously reported [46]. Briefly, after expression in E. coli BL21DE3, human CK2 alpha (aa. 1-336) was purified by sequential affinity, anion exchange and sizeexclusion chromatography (HiTrap Heparin, MonoQ and Superdex 75). Protein was concentrated to 
$10 \mathrm{mg} / \mathrm{ml}$ and frozen in liquid nitrogen. BRD4 first bromodomain was produced as described previously [47].

Crystals of apo CK2 and apo BRD4 and binding of inhibitors and fragments in their pockets were obtained as previously described [47-48]. Diffraction data were collected at the Elettra Synchrotron Light Source (Trieste, Italy), XRD1 beamline (PDB 6HOQ, 6HOR, 6HOT, 6HOU and 6HOV) and at the European Synchrotron Research Facility (ESRF, Grenoble, France), ID30-A3 beamline (PDB 6HOP). Data were processed with XDS [49] and Aimless [50]; structures were solved by molecular replacement with Phaser [51] using PDB 3Q04 [46] and 4IOO [47] as search models for CK2 and BRD4, respectively. Initial models were refined alternating cycles of automatic refinement with Phenix [52] and manual model building with COOT [53].

For the feruloylmethane, electron density does not allow distinguishing between the oxygen and the methyl of its acetyl moiety; however, a single pose has been assigned to the inhibitor for steric and energetic considerations.

For the curcumin-soaked structure, the protein chain was first fully refined and water molecules were added. Poses for ferulic acid, feruloylmethane, ferulic aldehyde and vanillin were imported from the relative structures in complex with CK2. Their occupancies were set to $25 \%$ and their Bfactors to $20 \AA^{2}$ and only these parameters were further refined (i.e. spatial coordinates were not allowed to be modified).

Data collection and refinement statistics are reported in Table S1.

Holoenzyme production, phosphorylation assays and kinetic determination

CK2 holoenzyme was purified as in [54] and incubated for 5-15 and $30 \mathrm{~min}$ at $37^{\circ} \mathrm{C}$ in a final volume of $25 \mu \mathrm{L}$ containing $50 \mathrm{mM}$ Tris $/ \mathrm{HCl}(\mathrm{pH} 7.5), 100 \mathrm{mM} \mathrm{NaCl}, 12 \mathrm{mM} \mathrm{MgCl} 2,100 \mu \mathrm{M}$ synthetic peptide substrate RRRADDSDDDDD (CK2-tide), 20 $\mu \mathrm{M} \quad\left[\gamma^{33} \mathrm{P}-\mathrm{ATP}\right] \quad$ (500$1000 \mathrm{cpm} / \mathrm{pmol}$ ), and different concentrations of the indicated inhibitors. Reaction was stopped by 
addition of $5 \mu \mathrm{L}$ of $0.5 \mathrm{M}$ orthophosphoric acid before spotting aliquots onto phosphocellulose filters.

Initial velocities were determined at each of the substrate concentrations tested. Km values were calculated either in the absence or presence of increasing concentrations of ferulic acid, from Lineweaver-Burk double-reciprocal plots of the data. Inhibition constant was then calculated by linear regression analysis of $\mathrm{Km} / \mathrm{Vmax}$ against inhibitor concentration plot.

\section{Cell culture, treatments and lysis}

Cells were cultured in an atmosphere containing $5 \% \mathrm{CO}_{2}$; Jurkat cells (human $\mathrm{T}$ lymphoblastoid cell line) were maintained in RPMI 1640 medium (Sigma), SK-N-BE and SH-SY5Y cells (human neuroblastoma cells) in D-MEM medium (Sigma); both media were supplemented with 10\% (v/v) fetal calf serum (FCS), 2mM L-glutamine, 100U/mL penicillin, and 100mg/mL streptomycin. Cell treatments with inhibitors were performed in the culture medium, but with $1 \%(\mathrm{v} / \mathrm{v})$ FCS. Control cells were treated with equal amounts of the inhibitor solvent, which never exceeded $1 \%(\mathrm{v} / \mathrm{v})$. For lysate preparation, cells were lysed as described in [33]. Protein concentration was determined by the Bradford method.

\section{Cell viability assay}

Cell viability was detected by means of MTT (3-(4,5-dimethylthiazol-2-yl)-3,5-diphenyltriazolium bromide) reagent: cells $\left(10^{5}\right.$ cells $\left./ 100 \mu \mathrm{l}\right)$ were incubated for variable times in a 96-well plate under the indicated conditions. $1 \mathrm{~h}$ before the end of the incubation, $10 \mu \mathrm{l}$ of MTT solution $(5 \mathrm{mg} / \mathrm{ml}$ in phosphate buffered saline) were added to each well. Incubations were stopped by addition of $20 \mu 1$ of lysis solution at $\mathrm{pH} 4.7$, as described elsewhere [33]. Plates were read for OD at $\lambda 590 \mathrm{~nm}$, in a Titertek Multiskan Plus plate reader (Flow Laboratories). 
Endocellular CK2 activity assay

CK2 activity of treated cells was measured by means of radioactive assays of lysates with $[\gamma$ $\left.{ }^{33} \mathrm{P}\right]$ ATP towards the specific CK2 substrate peptide CK2-tide, as described in [55].

Endocellular CK2 activity was also evaluated by assessing the phosphorylation state of the CK2 substrate Akt phosphor-Ser129 (Abcam) and anti cdc37 phospho-Ser13 (Abcam) as in [36]. For this purpose, equal amounts of proteins from treated cells were loaded on 11\% SDS-PAGE, blotted on Immobilon-P membranes (Millipore), and processed in Western blot (WB) with the indicated antibody, detected by chemiluminescence. Quantitation of the signal was obtained by chemiluminescence detection on a Kodak Image Station 440MMPRO and analysis with the Kodak 1DImage software. Signal was normalized to that obtained by WB with total Akt antibody and total cdc37 antibody (Santa Cruz Biotechnology), while loading control was provided by anti- $\beta$ actin antibody (Sigma-Aldrich).

\section{Accession Codes.}

Structures were deposited to the PDB with accession numbers 6HOP (CK2 $\alpha /$ curcumin), 6HOQ (CK2 $\alpha /$ ferulic acid), 6HOR (CK2 $\alpha /$ feruloylmethane), 6HOT (CK2 $\alpha /$ ferulic aldehyde), 6HOU (CK2 $\alpha /$ vanillin) and 6HOV (BRD4/ferulic acid).

\section{ASSOCIATED CONTENT}

The Supporting Information contains:

Table S1 (crystallographic data collection and refinement statistics).

\section{DECLARATION OF INTEREST}

The authors declare no competing financial interest. 


\section{ACKNOWLEDGMENTS}

We are grateful to the Structural Genomics Consortium (Oxford) for providing us with the expression vector for the N-terminal bromodomain of BRD4 and to the staff of the ID30-A3 and XRD1 beamlines at ESRF (Grenoble, France) and Elettra (Trieste, Italy), respectively, for on-site assistance. G.C. is supported by Fondazione Ricerca Fibrosi Cistica (FFC\#10/2017). G.L. is supported by the Associazione Italiana per la Ricerca sul Cancro (AIRC "MFAG 2017", code 19882).

\section{References}

1. Franchin, C., Borgo, C., Zaramella, S., Cesaro, L., Arrigoni, G., Salvi, M., and Pinna LA. (2017) Exploring the CK2 Paradox: Restless, Dangerous, Dispensable. Pharmaceuticals. 10, E11

2. Lolli, G., Pinna, L. A., and Battistutta, R. (2012) Structural determinants of protein kinase CK2 regulation by autoinhibitory polymerization. ACS Chem. Biol. 7, 1158-1163.

3. Lolli, G., Ranchio, A., and Battistutta, R. (2014) Active form of the protein kinase CK2 $\alpha 2 \beta 2$ holoenzyme is a strong complex with symmetric architecture. ACS Chem. Biol. 9, 366-371.

4. Lolli, G., Naressi, D., Sarno, S., and Battistutta, R. (2017) Characterization of the oligomeric states of the CK2 $\alpha_{2} \beta_{2}$ holoenzyme in solution. Biochem. J. 474, 2405-2416.

5. Gowda, C., Sachdev, M., Muthusami, S., Kapadia, M., Petrovic-Dovat, L., Hartman, M., Ding, Y., Song, C., Payne, J. L., Tan, B. H., and Dovat, S. (2017) Casein Kinase II (CK2) as a Therapeutic Target for Hematological Malignancies. Curr. Pharm. Des. 23, 95-107.

6. Piazza, F., Manni, S., Ruzzene, M., Pinna, L. A., Gurrieri, C., and Semenzato, G. (2012) Protein kinase CK2 in hematologic malignancies: reliance on a pivotal cell survival regulator by oncogenic signaling pathways. Leukemia 26, 1174-1179. 
7. Buontempo, F., McCubrey, J. A., Orsini, E., Ruzzene, M., Cappellini, A., Lonetti, A., Evangelisti, C., Chiarini, F., Evangelisti, C., Barata, J. T., and Martelli, A. M. (2018) Therapeutic targeting of CK2 in acute and chronic leukemias. Leukemia 32, 1-10.

8. Battistutta, R., Cozza, G., Pierre, F., Papinutto, E., Lolli, G., Sarno, S., O'Brien, S. E., SiddiquiJain, A., Haddach, M., Anderes, K., Ryckman, D. M., Meggio, F., and Pinna, L. A. (2011) Unprecedented selectivity and structural determinants of a new class of protein kinase CK2 inhibitors in clinical trials for the treatment of cancer. Biochemistry 50, 8478-8488.

9. https://www.cancer.gov/about-cancer/treatment/clinical-trials/intervention/silmitasertib-sodium

10. https://www.accessdata.fda.gov/scripts/opdlisting/oopd/detailedIndex.cfm?cfgridkey=548516

11. Kumar, G., Mittal, S., Sak, K., and Tuli, H. S. (2016) Molecular mechanisms underlying chemopreventive potential of curcumin: Current challenges and future perspectives. Life Sci. $148,313-328$.

12. Liu, S., Hsieh, D., Yang, Y. L., Xu, Z., Peto, C., Jablons, D. M., and You, L. (2013) Coumestrol from the national cancer Institute's natural product library is a novel inhibitor of protein kinase CK2. BMC Pharmacol. Toxicol. 14, 36.

13. Shen, L., and Ji, H. F. (2012) The pharmacology of curcumin: is it the degradation products? Trends Mol. Med. 18, 138-144.

14. Nelson, K. M., Dahlin, J. L., Bisson, J., Graham, J., Pauli, G. F., and Walters, M. A. (2017) The Essential Medicinal Chemistry of Curcumin. J. Med. Chem. 60, 1620-1637.

15. Baker, M. Deceptive curcumin offers cautionary tale for chemists. (2017) Nature 541, 144-145.

16. Heger, M. Drug screening: Don't discount all curcumin trial data. (2017) Nature 543, 40.

17. Mancuso, C., and Santangelo, R. (2014) Ferulic acid: pharmacological and toxicological aspects. Food Chem. Toxicol. 65, 185-195.

18. Lirdprapamongkol, K., Sakurai, H., Suzuki, S., Koizumi, K., Prangsaengtong, O., Viriyaroj, A., Ruchirawat, S., Svasti, J., and Saiki, I. (2010) Vanillin enhances TRAIL-induced apoptosis in cancer cells through inhibition of NF-kappaB activation. In Vivo 24, 501-506. 
19. Hampannavar, G. A., Karpoormath, R., Palkar, M. B., and Shaikh, M. S. (2016) An appraisal on recent medicinal perspective of curcumin degradant: Dehydrozingerone (DZG). Bioorg. Med. Chem. 24, 501-520.

20. Bruyère, C., Genovese, S., Lallemand, B., Ionescu-Motatu, A., Curini, M., Kiss, R., and Epifano, F. (2011) Growth inhibitory activities of oxyprenylated and non-prenylated naturally occurring phenylpropanoids in cancer cell lines. Bioorg. Med. Chem. Lett. 21, 4174-4179.

21. Filippakopoulos, P., and Knapp, S. (2014) Targeting bromodomains: epigenetic readers of lysine acetylation. Nat. Rev. Drug Discov. 13, 337-356.

22. www.clinicaltrials.gov

23. Marchand, J. R., Dalle Vedove, A., Lolli, G., and Caflisch A. (2017) Discovery of Inhibitors of Four Bromodomains by Fragment-Anchored Ligand Docking. J. Chem. Inf. Model. 57, 25842597.

24. Dutra, L.A., Heidenreich, D., Silva, G. D. B. D., Man Chin, C., Knapp, S., and Santos J. L. D. (2017) Dietary Compound Resveratrol Is a Pan-BET Bromodomain Inhibitor. Nutrients 9 (11).

25. Wu, S. Y., Lee, A. Y., Lai, H. T., Zhang, H., and Chiang, C. M. (2013) Phospho switch triggers Brd4 chromatin binding and activator recruitment for gene-specific targeting. Mol. Cell. 49, 843-857.

26. Lian, H., Li, D., Zhou, Y., Landesman-Bollag, E., Zhang, G., Anderson, N. M., Tang, K. C., Roderick, J. E., Kelliher, M. A., Seldin, D. C., Fu, H., and Feng, H. (2017) CK2 inhibitor CX4945 destabilizes NOTCH1 and synergizes with JQ1 against human T-acute lymphoblastic leukemic cells. Haematologica 102, e17-e21.

27. Korb, E., Herre, M., Zucker-Scharff, I., Gresack, J., Allis, C. D., and Darnell, R. B. (2017) Excess Translation of Epigenetic Regulators Contributes to Fragile X Syndrome and Is Alleviated by Brd4 Inhibition. Cell 170, 1209-1223.

28. Krissinel, E., and Henrick, K. (2007). Inference of macromolecular assemblies from crystalline state. J. Mol. Biol. 372, 774-797. 
29. Cozza, G., Mazzorana, M., Papinutto, E., Bain, J., Elliott, M., di Maira, G., Gianoncelli, A., Pagano, M. A., Sarno, S., Ruzzene, M., Battistutta, R., Meggio, F., Moro, S., Zagotto, G., and Pinna, L. A. (2009) Quinalizarin as a potent, selective and cell-permeable inhibitor of protein kinase CK2. Biochem. J. 421, 387-395.

30. Cozza, G., Venerando, A., Sarno, S., and Pinna, L. A. (2015) The Selectivity of CK2 Inhibitor Quinalizarin: A Reevaluation. Biomed. Res. Int. 2015, 734127.

31. Uhle, S., Medalia, O., Waldron, R., Dumdey, R., Henklein, P., Bech-Otschir, D., Huang, X., Berse, M., Sperling, J., Schade, R., and Dubiel, W. (2003) Protein kinase CK2 and protein kinase D are associated with the COP9 signalosome. EMBO J. 22, 1302-1312.

32. Franck, N., Le Seyec, J., Guguen-Guillouzo, C., and Erdtmann, L. (2005) Hepatitis C virus NS2 protein is phosphorylated by the protein kinase CK2 and targeted for degradation to the proteasome. J. Virol. 79, 2700-2708.

33. Zanin, S., Borgo, C., Girardi, C., O'Brien, S. E., Miyata, Y., Pinna, L. A., Donella-Deana, A., and Ruzzene, M. (2012) Effects of the CK2 inhibitors CX-4945 and CX-5011 on drug-resistant cells. PLoS One 7, e49193.

34. Siddiqui-Jain, A., Drygin, D., Streiner, N., Chua, P., Pierre, F., O'Brien, S. E., Bliesath, J., Omori, M., Huser, N., Ho, C., Proffitt, C., Schwaebe, M. K., Ryckman, D. M., Rice, W. G., and Anderes, K. (2010) CX-4945, an orally bioavailable selective inhibitor of protein kinase CK2, inhibits prosurvival and angiogenic signaling and exhibits antitumor efficacy. Cancer Res. 70, 10288-10298.

35. Füllbeck, M., Huang, X., Dumdey, R., Frommel, C., Dubiel, W., and Preissner, R. (2005) Novel curcumin- and emodin-related compounds identified by in silico 2D/3D conformer screening induce apoptosis in tumor cells. BMC Cancer. 5, 97.

36. Di Maira, G., Brustolon, F., Pinna, L. A., and Ruzzene, M. (2009) Dephosphorylation and inactivation of Akt/PKB is counteracted by protein kinase CK2 in HEK 293T cells. Cell. Mol. Life Sci. 66, 3363-3373. 
37. Miyata, Y., and Nishida, E. (2005) CK2 binds, phosphorylates, and regulates its pivotal substrate Cdc37, an Hsp90-cochaperone. Mol. Cell. Biochem. 274, 171-179.

38. Battistutta, R., Mazzorana, M., Cendron, L., Bortolato, A., Sarno, S., Kazimierczuk, Z., Zanotti, G., Moro, S., and Pinna L. A. (2007) The ATP-binding site of protein kinase CK2 holds a positive electrostatic area and conserved water molecules. Chembiochem 8, 1804-1809.

39. Hopkins, A. L., Keserü, G. M., Leeson, P. D., Rees, D. C., and Reynolds, C. H. (2014) The role of ligand efficiency metrics in drug discovery. Nat. Rev. Drug Discov. 13, 105-121.

40. Murray, C. W., Erlanson, D. A., Hopkins, A. L., Keserü, G. M., Leeson, P. D., Rees, D. C., Reynolds, C. H., and Richmond, N. J. (2014) Validity of ligand efficiency metrics. ACS Med. Chem. Lett. 5, 616-618.

41. Ghosh, S., Basak, P., Dutta, S., Chowdhury, S., and Sil, P. C. (2017) New insights into the ameliorative effects of ferulic acid in pathophysiological conditions. Food Chem. Toxicol. 103, $41-55$.

42. Yang, G. W., Jiang, J. S., and Lu, W. Q. (2015) Ferulic Acid Exerts Anti-Angiogenic and AntiTumor Activity by Targeting Fibroblast Growth Factor Receptor 1-Mediated Angiogenesis. Int. J. Mol. Sci. 16, 24011-24031.

43. Cozza, G., Zanin, S., Sarno, S., Costa, E., Girardi, C., Ribaudo, G., Salvi, M., Zagotto, G., Ruzzene, M., and Pinna, L. A. (2015) Design, validation and efficacy of bisubstrate inhibitors specifically affecting ecto-CK2 kinase activity. Biochem. J. 471, 415-430.

44. Montenarh, M., and Götz, C. (2018) Ecto-protein kinase CK2, the neglected form of CK2. Biomed. Rep. 8, 307-313.

45. Zylbersztejn, A. M., de Morais, C. G., Lima, A. K., Souza, J. E., Lopes, A. H., Da-Silva, S. A., Silva-Neto, M. A., and Dutra, P. M. (2015) CK2 Secreted by Leishmania braziliensis Mediates Macrophage Association Invasion: A Comparative Study between Virulent and Avirulent Promastigotes. Biomed. Res. Int. 2015, 167323. 
46. Papinutto, E., Ranchio, A., Lolli, G., Pinna, L. A., and Battistutta, R. (2012) Structural and functional analysis of the flexible regions of the catalytic $\alpha$-subunit of protein kinase CK2. $J$. Struct. Biol. 177, 382-391.

47. Lolli, G., and Battistutta, R. (2013) Different orientations of low-molecular-weight fragments in the binding pocket of a BRD4 bromodomain. Acta Crystallogr. D Biol. Crystallogr. 69, 21612164.

48. Cozza, G., Girardi, C., Ranchio, A., Lolli, G., Sarno, S., Orzeszko, A., Kazimierczuk, Z., Battistutta, R., Ruzzene, M., and Pinna, L. A. (2014) Cell-permeable dual inhibitors of protein kinases CK2 and PIM-1: structural features and pharmacological potential. Cell. Mol. Life Sci. $71,3173-3185$.

49. Kabsch, W. (2010) XDS. Acta Crystallogr. D Biol. Crystallogr. 66, 125-132.

50. Evans, P. R., and Murshudov, G. N. (2013) How good are my data and what is the resolution? Acta Crystallogr. D Biol. Crystallogr. 69, 1204-1214.

51. McCoy, A. J., Grosse-Kunstleve, R. W., Adams, P. D., Winn, M. D., Storoni, L. C., and Read, R. J. (2007) Phaser crystallographic software. J. Appl. Crystallogr. 40, 658-674.

52. Adams, P. D., Afonine, P. V., Bunkóczi, G., Chen, V. B., Davis, I. W., Echols, N., Headd, J. J., Hung, L.-W., Kapral, G. J., Grosse-Kunstleve, R. W., McCoy, A. J., Moriarty, N. W., Oeffner, R., Read, R. J., Richardson, D. C., Richardson, J. S., Terwilliger, T. C., and Zwart, P. H. (2010) PHENIX: a comprehensive Python-based system for macromolecular structure solution. Acta Crystallogr. D Biol. Crystallogr. 66, 213-221.

53. Emsley, P., Lohkamp, B., Scott, W. G., and Cowtan, K. (2010) Features and development of Coot. Acta Crystallogr. D Biol. Crystallogr. 66, 486-501.

54. Venerando, A., Franchin, C., Cant, N., Cozza, G., Pagano, M. A., Tosoni, K., Al-Zahrani, A., Arrigoni, G., Ford, R. C., Mehta, A., and Pinna, L. A. (2013) Detection of phospho-sites generated by protein kinase CK2 in CFTR: mechanistic aspects of Thr1471 phosphorylation, PLoS One 8, e74232. 
55. Ruzzene, M., Di Maira, G., Tosoni, K., and Pinna, L. A. (2010) Assessment of CK2 constitutive activity in cancer cells. Methods Enzymol. 484, 495-514.

\section{Graphical Abstract}

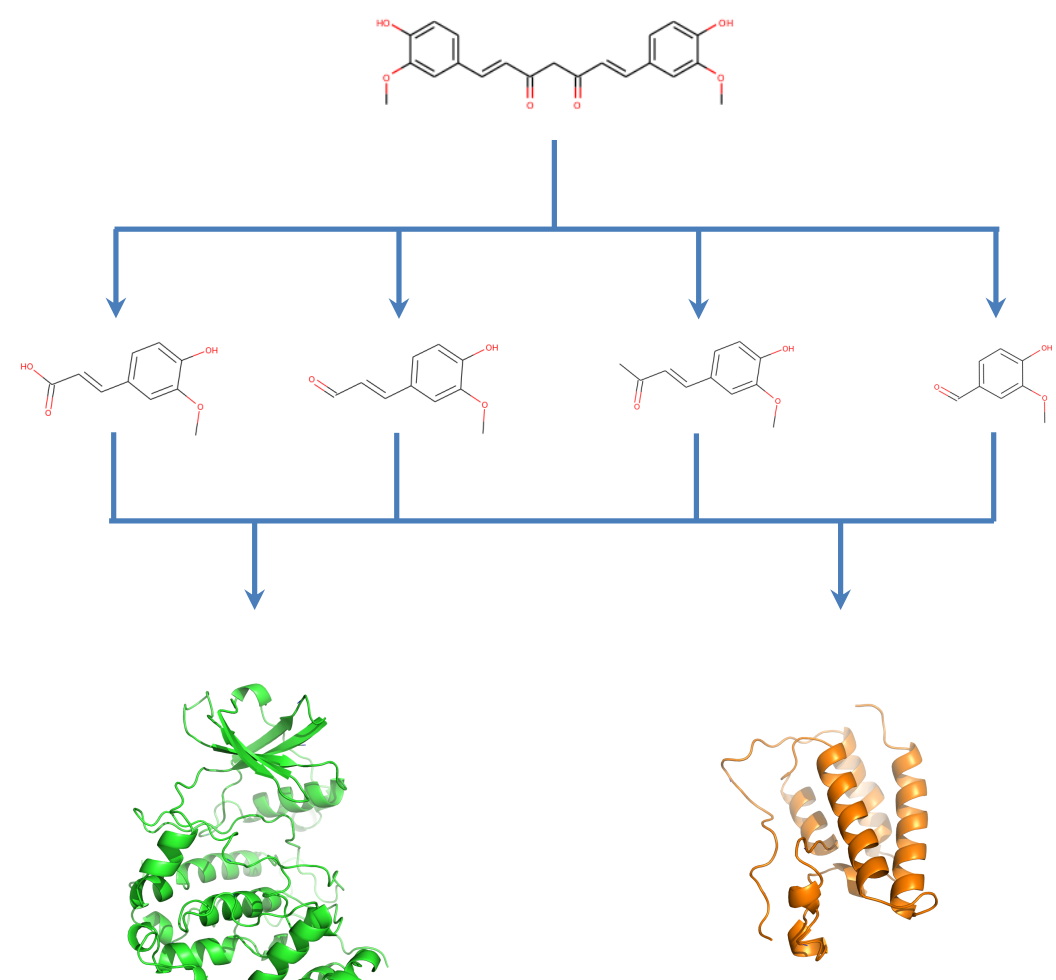

